\title{
Analysis of Arbitrarily Shaped Dielectric Lens Antenna by Ray Tracing Method
}

\author{
Mitsuo TAGUCHI*, Mami IGASAKI**, Hideaki SHIMODA*** and Kazumasa TANAKA \\ Dept. of Electrical \& Electronic Eng., Nagasaki University \\ ** Graduate School of Science and Technology, Nagasaki University \\ 1-14 Bunkyo-machi, Nagasaki-shi, 852-8521, Japan \\ *** Telecom Technology Development Center, TDK Co. \\ 2-15-7, Higashi-Ohwada, Ichikawa-shi, Chiba, 272-0026 Japan \\ E-mail: mtaguchi@net.nagasaki-u.ac.jp
}

\section{Introduction}

Since a dielectric lens antenna has narrow beamwidth, low side lobes and scanning capability, it is considered as the promising candidate for the antenna of future mobile communication systems and ITS services [1]. The ray tracing method is used to estimate the radiation characteristics of lens antenna [2], [3]. In this paper, the ray tracing method is applied to the lens antenna with arbitrarily shaped inner and outer surfaces.

\section{Formulation}

Figure 1 shows the dielectric lens antenna and the coordinate system for the ray tracing method. Let the source point on the primary radiator be $P_{0}\left(x_{0}, y_{0}, z_{0}\right)$. The equivalent electric and magnetic currents $J_{y}\left(x_{0}, y_{0}\right) \boldsymbol{i}_{y}$ and $M_{x}\left(x_{0}, y_{0}\right) \boldsymbol{i}_{x}$ are assumed on the source point $P_{0} . \quad \boldsymbol{i}_{x}, \boldsymbol{i}_{y}$ and $\boldsymbol{i}_{z}$ are the unit vectors of the Cartesian coordinate system. The inner surface of lens is $S_{1}$ and the outer surface of lens is $S_{2}$ and the reference plane of lens antenna is $S_{3}$. The points of the ray on $S_{1}, S_{2}$ and $S_{3}$ are defined as $P_{1}\left(x_{1}, y_{1}, z_{1}\right)$, $P_{2}\left(x_{2}, y_{2}, z_{2}\right)$ and $P_{3}\left(x_{3}, y_{3}, z_{3}\right)$, respectively.

Snell's law is expressed as follows:

$$
k_{1} \sin \theta_{1}=k_{2} \sin \theta_{2}
$$

where $k_{1}$ and $k_{2}$ are the propagation constants within the outside and inside region of lens, and $\theta_{1}$ and $\theta_{2}$ are the incident and the refractive angles, respectively. Applying Eq. (1) to the ray tracing method, the points $P_{1}, P_{2}$ and $P_{3}$ on $S_{1}, S_{2}$ and $S_{3}$ can be obtained straightforwardly.

Fresnel's transmission coefficients of parallel and perpendicular polarization are given by the following expressions, respectively.

$$
\begin{aligned}
T_{T M} & =\frac{2 \mu_{2} n \cos \theta_{1}}{\mu_{1} n^{2} \cos \theta_{1}+\mu_{2} \sqrt{n^{2}-\sin ^{2} \theta_{1}}} \\
T_{T E} & =\frac{2 \mu_{2} \cos \theta_{1}}{\mu_{2} \cos \theta_{1}+\mu_{1} \sqrt{n^{2}-\sin ^{2} \theta_{1}}}
\end{aligned}
$$

where $n$ is the refractive index of lens.

$$
n=\frac{k_{2}}{k_{1}}=\sqrt{\frac{\mu_{2}}{\mu_{1}}} \sqrt{\frac{\varepsilon_{2}-j\left(\sigma_{2} / \omega\right)}{\varepsilon_{1}-j\left(\sigma_{1} / \omega\right)}}
$$

By using eqs. (1) - (4), the incident electric field at $P_{1}$ is expressed as follows. 


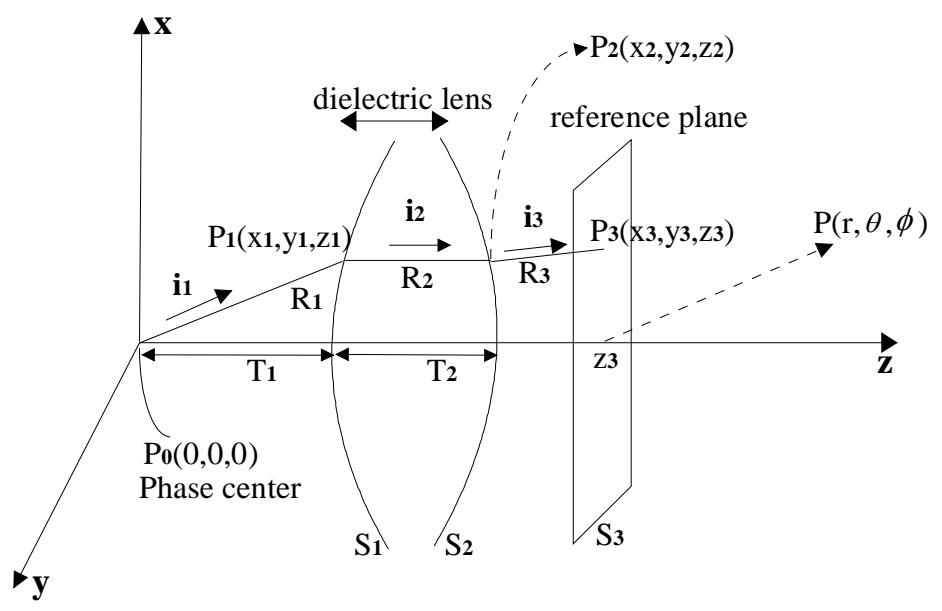

Figure 1. Coordinate system for ray tracing method.

$$
\begin{gathered}
\boldsymbol{E}_{P_{1}}^{\text {in }}=\left(E_{P_{1}}^{x} \boldsymbol{i}_{x}+E_{P_{1}}^{y} \boldsymbol{i}_{y}+E_{P_{1}}^{z} \boldsymbol{i}_{z}\right) \psi \\
E_{P_{1}}^{x}\left(x_{1}, y_{1}\right)=\frac{k_{1}^{2}}{4 \pi R_{1}^{2}}\left(x_{1}-x_{0}\right)\left(y_{1}-y_{0}\right) J_{y}\left(x_{0}, y_{0}\right) \\
E_{P_{1}}^{y}\left(x_{1}, y_{1}\right)=\frac{1}{4 \pi}\left[\left\{\frac{k_{1}^{2}}{R_{1}^{2}}\left(y_{1}-y_{0}\right)^{2}-j \omega \mu_{0}\right\} J_{y}\left(x_{0}, y_{0}\right)+\frac{j k_{1}}{R_{1}}\left(z_{1}-z_{0}\right) M_{x}\left(x_{0}, y_{0}\right)\right] \\
E_{P_{1}}^{z}\left(x_{1}, y_{1}\right)=\frac{1}{4 \pi}\left\{\frac{k_{1}^{2}}{R_{1}^{2}}\left(y_{1}-y_{0}\right)\left(z_{1}-z_{0}\right) J_{y}\left(x_{0}, y_{0}\right)-\frac{j k_{1}}{R_{1}}\left(y_{1}-y_{0}\right) M_{x}\left(x_{0}, y_{0}\right)\right\} \\
\psi=\frac{\exp \left(-j k R_{1}\right)}{R_{1}}
\end{gathered}
$$

The electric field on the reference plane $S_{3}$ is obtained as,

$$
\begin{gathered}
\boldsymbol{E}_{P_{3}}=\left(E_{P_{3}}^{x} \boldsymbol{i}_{x}+E_{P_{3}}^{y} \boldsymbol{i}_{y}+E_{P_{3}}^{z} \boldsymbol{i}_{z}\right) \psi \\
E_{P_{3}}^{i}\left(x_{3}, y_{3}\right)=\left\{K_{x}^{i} E_{P_{1}}^{x}\left(x_{1}, y_{1}\right)+K_{y}^{i} E_{P_{1}}^{y}\left(x_{1}, y_{1}\right)+K_{z}^{i} E_{P_{1}}^{z}\left(x_{1}, y_{1}\right)\right\} \\
\times \frac{\exp \left\{-j\left(k_{1} R_{1}+k_{2} R_{2}+k_{1} R_{3}\right)\right\}}{R_{1} R_{2} R_{3}}, \quad i=x, y, \text { or } z \\
R_{1}=\overline{P^{\prime} P_{1}}, \quad R_{2}=\overline{P_{1} P_{2}}, \quad R_{3}=\overline{P_{2} P_{3}}
\end{gathered}
$$

$K_{x}^{i}, K_{y}^{i}, K_{z}^{i}$ denote the variables defined by the normal vectors and the transmission coefficients at the points $P_{1}$ and $P_{2}$. The transmission coefficient at each point depends on the mode of incident ray.

The radiated field is calculated by the surface integral on the reference plane $S_{3}$. Replacing the coordinate origin to the point of intersection of $S_{3}$ and the z-axis, the electric field is expressed by

$$
\begin{aligned}
\boldsymbol{E}(r, \theta, \phi) & =\frac{1}{4 \pi} \int_{S_{3}}\left\{-j \omega \mu \psi\left(\boldsymbol{n}^{\prime} \times \boldsymbol{H}\right)+\left(\boldsymbol{n}^{\prime} \times \boldsymbol{E}\right) \times \nabla^{\prime} \psi+\left(\boldsymbol{n}^{\prime} \cdot \boldsymbol{E}\right) \nabla^{\prime} \psi\right\} d S^{\prime} \\
& =\frac{\exp (-j k r)}{r} \boldsymbol{D}(\theta, \phi)
\end{aligned}
$$



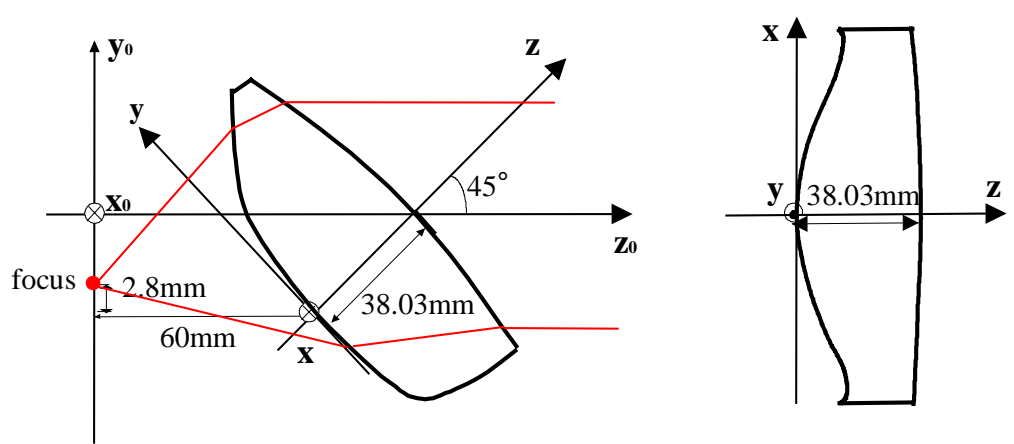

(a) cross sectional view

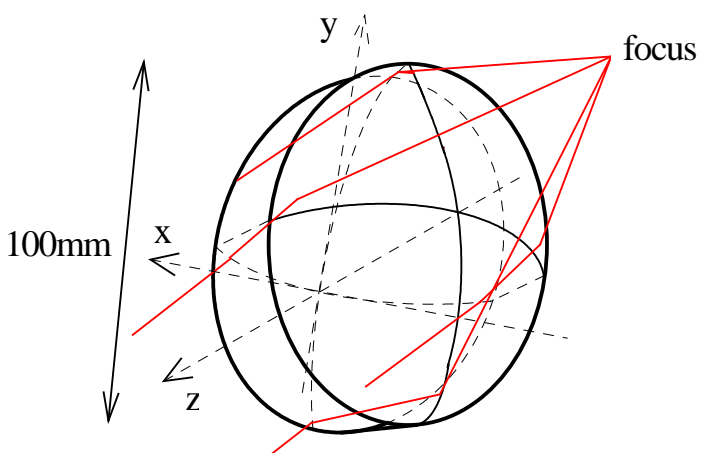

(b) three-dimensional view

Figure 2. Geometry of dielectric lens antenna.

$$
\begin{array}{r}
\boldsymbol{D}(\theta, \phi)=\frac{j k_{1}}{4 \pi} \int_{S_{3}}\left[\left\{E_{P_{3}}^{x}\left(x_{3}, y_{3}\right) \cos \phi+E_{P_{3}}^{y}\left(x_{3}, y_{3}\right) \sin \phi\right\} \boldsymbol{i}_{\theta}-\left\{E_{P_{3}}^{x}\left(x_{3}, y_{3}\right) \cos \theta \sin \phi\right.\right. \\
\left.\left.-E_{P_{3}}^{y}\left(x_{3}, y_{3}\right) \cos \theta \cos \phi\right\} \boldsymbol{i}_{\phi}\right] \exp \left\{j k_{1}\left(x_{3} \sin \theta \cos \phi+y_{3} \sin \theta \sin \phi\right)\right\} d S^{\prime}
\end{array}
$$

\section{Numerical and experimental results}

Figure 2 shows the geometry of the dielectric lens for the numerical analysis. The primary radiator is the conical horn antenna fed by the rectangular waveguide. The phase center of primary radiator is located at the focus of lens $(0,-24.09 \mathrm{~mm}, 0)$. The dielectric lens is formed from a circular cylinder with the diameter of $100 \mathrm{~mm}$. The inner surface of lens is unsymmetrical. If the lens surfaces are given by numerical discrete data, the radiation characteristics is calculated by the ray tracing method. In the example shown in Figure 2, the geometry of inner surface of dielectric lens is expressed by the following equation.

$$
\begin{aligned}
z= & C_{0,1} x^{0} y^{1}+\cdots+C_{0,8} x^{0} y^{8}+C_{2,0} x^{2} y^{0}+\cdots+C_{2,6} x^{2} y^{6}+C_{4,0} x^{4} y^{0} \\
& +\cdots+C_{4,4} x^{4} y^{4}+C_{6,0} x^{6} y^{0}+\cdots+C_{6,2} x^{6} y^{2}+C_{8,0} x^{8} y^{0}
\end{aligned}
$$

The curvature radii of outer surface of dielectric lens are $1000 \mathrm{~mm}$ in the xz-plane and $200 \mathrm{~mm}$ in the yz-plane. The lens is made of ABS resin. The relative permittivity of ABS resin is 2.641 at the frequency of $76.5 \mathrm{GHz}$.

Figure 3 shows the electric field radiation patterns of lens antenna at the frequency of 76.5 GHz. The calculated half-power beamwidth in the E-plane is 3.6 degrees, and the measured one is 3.3 degrees. In the H-plane, the calculated half-power beamwidth is 3.25 degrees, and the measured one is 2.9 degrees. 


\section{Conclusion}

The ray tracing method is employed to calculate the radiation characteristics of arbitrarily shaped dielectric lens antenna. As an example of lens antenna, the circular cylindrical lens antenna with an inner surface of unsymmetrical shape is calculated. The calculated half-power beamwidth agrees well with the measured result. In the next step, we will design and calculate the lens antenna for the mobile communication system.

\section{References}

[1] N. Kitamori et al., "High- $\varepsilon$ ceramic lens antenna with novel beam scanning mechanism", Proc. of ISAP2000, pp.983-986, Aug. 2000.

[2] M. Taguchi, M. Masuda, H. Shimoda and K. Tanaka, "Analysis of arbitrarily shaped dielectric lens antenna”, Proc. of IEEE AP-S Int. Symp., vol.2, pp.769-772, July 2001.

[3] Y. Yamada and S. Sasaki, "Estimations of radiation characteristics of an off-forcus feed shaped dielectric lens antenna by a ray tracing method", Proc. of IEEE AP-S Int. Symp., vol.4, pp.398-401, June 2002.

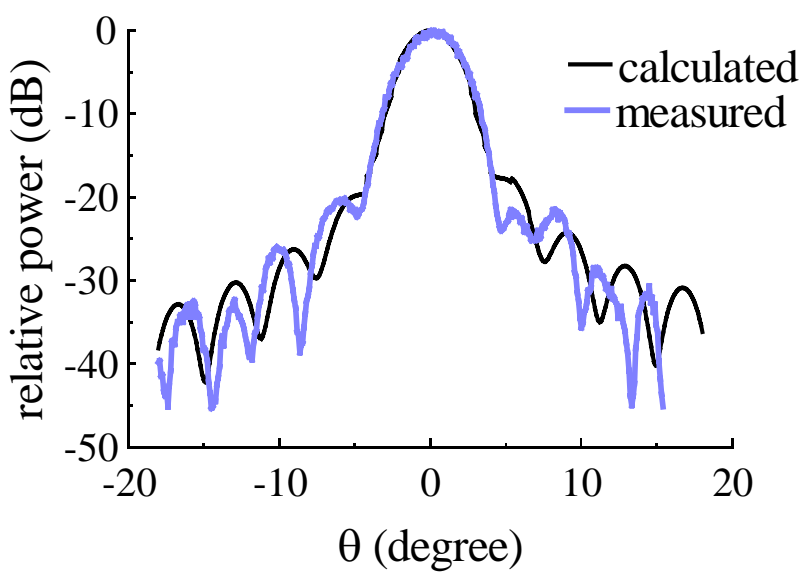

(a) E-plane patterns

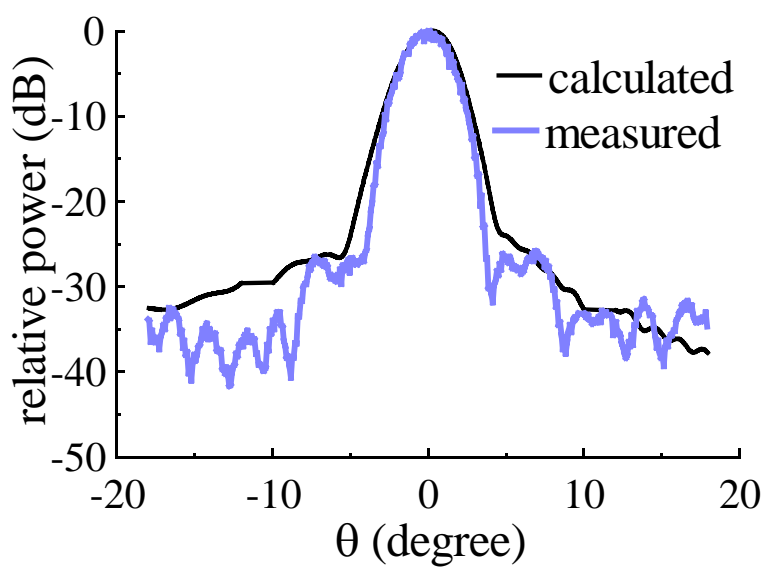

(b) H-plane patterns

Figure 3. Electric field radiation patterns of dielectric lens antenna at $76.5 \mathrm{GHz}$. 